\title{
Vitamin D as Nutritional Strategy to Treat Autism
}

Afaf El-ansary ${ }^{1 *}$ and Hanan Qasem ${ }^{2}$

${ }^{1}$ Central Laboratory, Center for Female Scientific and Medical Colleges at King Saud University, Saudi Arabia

${ }^{2}$ Autism Research and Treatment Center, King Saud University, Saudi Arabia

*Corresponding author: Afaf El-ansary, Central Laboratory, Center for Female Scientific and Medical Colleges at King Saud University; Riyadh, Saudi Arabia

Submission: February 23, 2018; Published: March 27, 2018

\section{Letter to the Editor}

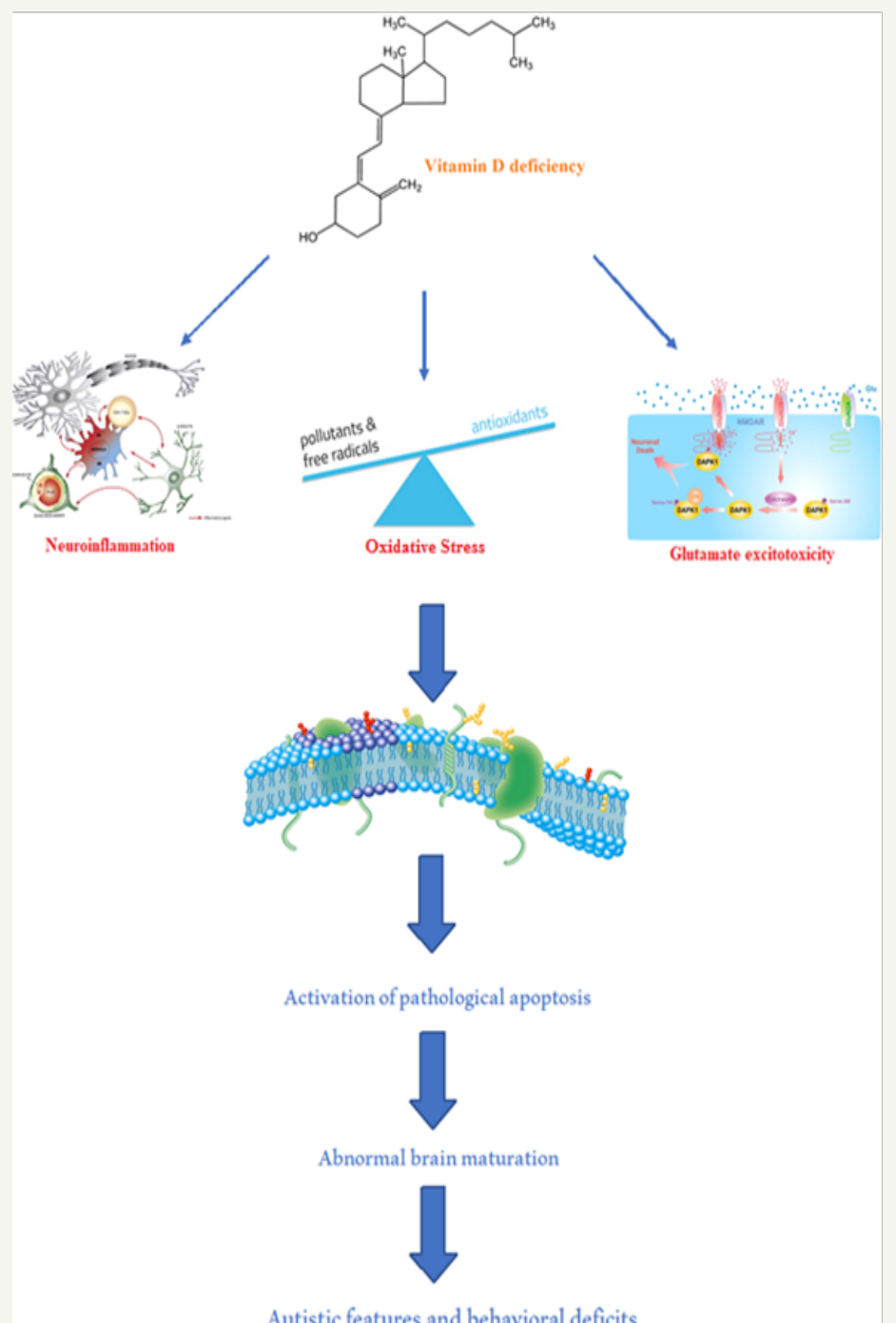

Figure 1: Demonstrates the suggested relationship between vitamin deficiency and neuro inflammation, oxidative stress and glutamate excite toxicity. It is Illustration of the relationship between vitamin D deficiency and neuro inflammation, oxidative stress, and glutamate excite toxicity as three etiological mechanisms in autism).

Autism is a neuro developmental disorder that is caused by many factors such as genetics, environment and maternal condition. There are a lot of environmental risk factors that influence autism pathogenesis by their epigenetic effects [1]. Among the risk factors of autism as a multifactorial disorder is the low vitamin D status in early development. The concurrent increase of prevalence of 
vitamin D deficiency and autism during the last 20 years might help to suggest vitamin D deficiency/insufficiency during pregnancy or in the first year of life as a contributor which can be related to different etiological mechanisms of autism. Confirmation of the role of vitamin D status in autism may provide strategy for primary and secondary preventive interventions. This manuscript is an attempt to solve the contradiction related to the role of vitamin $\mathrm{D}$ deficiency/insufficiency in autism and to find its relationship with oxidative stress; neuro inflammation, autoimmunity and glutamate excite toxicity and apoptosis as pathos-physiological mechanisms. It is well known that iron and zinc-induced oxidative stress in rat brain through depletion of glutathione and subsequent generation of reactive oxygen and nitrogen inflammatory species can be ameliorated by activated vitamin D. Repletion of glutathione by vitamin D can be easily related to detoxification of mercury and lead heavy metal burden repeatedly reported in individuals with autism as poor detoxifiers [2-4].

Based on the fact that pro-inflammatory cytokines and chemokines such as MCP-1, TNF- $\alpha$, IFN- $\Upsilon$, IL-6, and IL-12, are consistently elevated in individuals with autism [5-7], serum levels of vitamin D is significantly lower in autistic patients $[8,9]$, and that vitamin $\mathrm{D}$ has a profound anti-inflammatory action, so supplementation with vitamin D can help to reduce the neuroinflammatory insult in autism $[8,10]$. Anti-inflammatory action of vitamin $\mathrm{D}$ is also demonstrated as inhibition of the synthesis and biological effects of prostaglandins as inflammatory lipid mediator and NF- $\kappa B$, which is involved in aberrant signaling in autistic brains [11,12]. A significantly elevated levels of anti-myelinassociated glycoprotein (anti-MAG) auto-antibodies were found in children with autism and were negatively correlated with vitamin D levels, showing the relationship between vitamin D deficiency/ insufficiency and autoimmunity as etiological mechanism in autism $[13,14]$.

Glutamate excito toxicity as etiological mechanism in autism is due to overstimulation of glutamate receptors and down regulation of glutamate transporters, as proteins critically needed for the clearance of the excitatory neurotransmitter, glutamate from the synaptic cleft $[15,16]$. Based on our understanding of glutamate signaling in children with autism and the recently reported enhancement of glutamate transporter (EAAT3) expression by activated vitamin D as the legend for the Vitamin D Receptor (VDR), vitamin D supplementation can be of remarkable help in the regulation of the excito toxic glutamate handling in autism [17]. Using animal modeling of autism, Al-Fawaz 2014 were able to show the protective effects of vitamin D through the remarkable amelioration of the impaired biochemically measured parameters representing neuro chemical (Serotonin), inflammation (IFN-Y) and detoxification processes (Glutathione-s-transferase) [18].

As vitamin D deficiency in autistic children is confirmed, randomized controlled trials are urgently needed. A recent randomized controlled trial, published in the Journal of Child Psychiatry and Psychology, found that high dose vitamin D (300 IU/ $\mathrm{KG} /$ day) had a significant treatment effect on the core symptoms of autism [8]. In a more recent trial, Cannell 2017 reported that younger children and those children whose final vitamin D level exceeded $40 \mathrm{ng} / \mathrm{ml}$ responded best to vitamin D supplementation. If these trials are replicated, it would make the early diagnosis of autism very critical, because effective treatment with vitamin D may be possible [19] (Figure 1).

\section{References}

1. Padideh K, Elahe K, Seyyed MM, Mojgan K (2017) Environmental factors influencing the risk of autism. J Res Med Sci 22: 27.

2. John M (2016) Urine total glutathione levels as a potential marker of increased oxidative stress in autism. Pediatric Review: International Journal of Pediatric Research 3: 8.

3. Oshodi Y, Ojewunmi O, Oshodi TA, Ijarogbe GT, Ogun OC, et al. (2017) Oxidative stress markers and genetic polymorphisms of glutathione S-transferase T1, M1, and P1 in a subset of children with autism spectrum disorder in Lagos, Nigeria. Niger J Clin Pract 20(9): 1161-1167.

4. Alabdali A, Al-Ayadhi L, El-Ansary A (2014) Association of social and cognitive impairment and biomarkers in autism spectrum disorders. J Neuroinflammation 11: 4.

5. Xie J (2017) Immunological cytokine profiling identifies TNF-alfa as a key molecule dysregulated in autistic children. Oncotarget 8(47): 82390-82398.

6. Bryn V, Aass HC, Skjeldal OH, Isaksen J, Saugstad OD, et al. (2017) Cytokine Profile in Autism Spectrum Disorders in Children. J Mol Neurosci 61(1): 1-7.

7. El-Ansary A, Al-Ayadhi L (2012) Neuro inflammation in autism spectrum disorders. J Neuroinflammation 9(1): 265.

8. Saad K, Abdel-Rahman AA, Elserogy YM, Al-Atram AA, Cannell JJ, et al. (2016) Vitamin D status in autism spectrum disorders and the efficacy of vitamin D supplementation in autistic children. Nutritional neuroscience 19(8): 346-351.

9. Bener A, Khattab AO, Bhugra D, Hoffmann GF (2017) Iron and vitamin D levels among autism spectrum disorders children. Ann Afr Med 16(4): 186-191.

10. Endres D, Rick D, Oliver S, Armin B, Evgeniy P, et al. (2016) Vitamin D deficiency in adult patients with schizophreniform and autism spectrum syndromes: A one-year cohort study at a german tertiary care hospital. Front Psychiatry 7: 168.

11. Jeffery L (2016) 34 Anti-inflammatory effects of vitamin D are reduced in T-cells from the inflamed joints of rheumatoid arthritis patients, BMJ Publishing Group, UK.

12. De Vries MA, Van der Meulen N, van de Geijn GM, Klop B, Van Der Zwan EM, et al. (2017) Effect of a Single Dose of Vitamin D3 on Postprandial Arterial Stiffness and Inflammation in Vitamin D-Deficient Women. J Clin Endocrinol Metab 102(3): 992-1000.

13. Krakowiak P, Walker CK, Tancredi D, Hertz-Picciotto, Van de Water J, et al. (2017) Autism, Äêspecific maternal anti Äêfetal brain autoantibodies are associated with metabolic conditions. Autism Res 10(1): 89-98.

14. Mostafa GA, Laila Al-Ayadhi (2012) The relationship between the increased frequency of serum antineuronal antibodies and the severity of autism in children. European Journal of Paediatric Neurology 16(5): 464-468.

15. El-Ansary A (2016) Data of multiple regressions analysis between selected biomarkers related to glutamate excitotoxicity and oxidative stress in Saudi autistic patients. Data Brief 7: 111-116.

16. Essa MM, Braidy N, Vijayan KR, Subash S, Guillemin GJ, et al. (2013) Excitotoxicity in the pathogenesis of autism. Neurotox Res Springer 1267-1271. 
17. Beaudin S, Welsh J (2017) 1, 25-Dihydroxyvitamin D regulation of glutamine synthetase and glutamine metabolism in human mammary epithelial cells. Endocrinology 158(12): 4174-4188.

18. Alfawaz HA, Bhat RS, Al-Ayadhi L, El-Ansary AK (2014) Protective and restorative potency of Vitamin $\mathrm{D}$ on persistent biochemical autistic features induced in propionic acid-intoxicated rat pups. BMC Complement Altern Med 14(1): 416.

19. Cannell JJ (2017) Vitamin D and autism, what's new? 18(2): 183-193. 460-466.
(C) (1) Creative Commons Attribution 4.0

For possible submissions Click Here
Submit Article

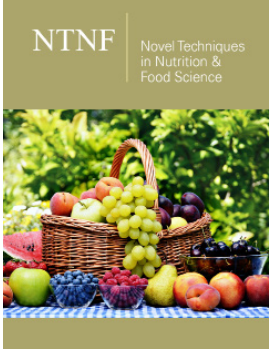

\section{Novel Techniques in Nutrition and Food Science}

\section{Benefits of Publishing with us}

- High-level peer review and editorial services

- Freely accessible online immediately upon publication

- Authors retain the copyright to their work

- Licensing it under a Creative Commons license

- Visibility through different online platforms 